\title{
InGaN/GaN nanowire based opto-chemical sensor for detecting hydrogen and hydrocarbons at low temperature
}

\author{
Sumit Paul ${ }^{1,2}$, Andreas Helwig ${ }^{1}$, Gerhard Müller ${ }^{1}$, Florian Furtmayer ${ }^{2}$, Jörg Teubert' ${ }^{2}$, Martin Eickhoff ${ }^{2}$ \\ ${ }^{1}$ EADS Innovation Works, 81663 Munich, Germany \\ ${ }^{2}$ I. Physikalisches Institut, Justus-Liebig-Universität, Heinrich-Buff-Ring 16, 35392 Gießen, Germany \\ Andreas.helwig@eads.net
}

\begin{abstract}
:
We report on InGaN/GaN nanowires (NWs) as opto-chemical transducers for the detection of $\mathrm{H}_{2}$ and hydrocarbons. The NWs exhibit a strong photoluminescence $(\mathrm{PL})$ which persists up to temperatures of $150^{\circ} \mathrm{C}$ and above. The GaN/InGaN NWs were grown by plasma-assisted molecular beam epitaxy on low-resistivity n-type $\mathrm{Si}(111)$ substrates and covered with a semitransparent catalytic Pt-coating after deposition. With the thin Pt coating on top, the PL intensity is dependent on the concentration of hydrogen or hydrocarbons in the surrounding atmosphere. Contrary to commercially available hydrogen sensors e.g. Pd or Pt gated high electron mobility transistors or catalytically activated metaloxide-silicon-carbide FETs our all-optical sensor system allows detection of ppm concentrations of $\mathrm{H}_{2}$ at low operating temperatures $\left(30^{\circ} \mathrm{C}-120^{\circ} \mathrm{C}\right)$.
\end{abstract}

Key words: nanosensors, $\mathrm{H}_{2}$, hydrocarbon, photoluminescence, nanowires, GaN, InGaN

\section{Background}

The detection of $\mathrm{H}_{2}$ and hydrocarbon gases plays an important role in diverse applications like leak detection in fuel and hydraulic lines, fire detection, etc. It has been demonstrated a long time ago that $\mathrm{H}_{2}$ and hydrocarbons can be detected using silicon and silicon carbide-based MOS devices [1,3]. More recent work has shown that field effect gas sensors can also be made from III - nitride materials [3]. These electronic devices respond well to $\mathrm{H}_{2}$, and in particular to hydrocarbons, only at temperatures well above room temperature (RT). We have utilized III-nitride based nanowire materials as opto-chemical transducers at RT. GaN/InGaN NWs exhibit an efficient PL at and above RT, which becomes chemically sensitive when the NWs are coated with thin $(5 \mathrm{~nm})$ catalytic $\mathrm{Pt}$ films [4]. The physics behind the sensing mechanism is the dissociation of molecular hydrogen on the Pt surface, diffusion of atomic hydrogen through the Pt film and formation of an $\mathrm{O}-\mathrm{H}$ dipole layer at the Pt-oxidised III-nitride surfaces. The result of such surface potential changes is a shift in the peak emission wavelength of the PL as well as a change in the PL intensity.

A key advantage of this opto-chemical transducer effect is that it provides a built-in media separation. This media separation arises from the fact that the GaN/InGaN NWs can be illuminated through mechanically strong and optically transparent sapphire substrates and that the PL can be excited and detected through these optically transparent windows without brining electronics in contact with the medium of interest.

\section{Design}

The NW transducer structures consist of a 300 $\mathrm{nm}$ GaN base part followed by a $100 \mathrm{~nm}$ InGaN-section. Fig. 1 presents a schematic of the NW structure and a scanning electron microscopy image of a NW ensemble. NW diameters vary between $25 \mathrm{~nm}$ and $50 \mathrm{~nm}$ and the NW heights are in the range between 350 $\mathrm{nm}$ and $400 \mathrm{~nm}$. $\ln _{\mathrm{x}} \mathrm{Ga}_{1-\mathrm{x}} \mathrm{N}$ with a suitable $\mathrm{In}$ content $x$ had been chosen as a NW material to obtain light emission at wavelengths longer than $450 \mathrm{~nm}$ for attaining a larger Stokes shift in the sensor system. Low resistivity n-type $\mathrm{Si}$ (111) substrates were used. The substrates were exposed to nitrogen plasma at the growth temperature of $780^{\circ} \mathrm{C}$ for $15 \mathrm{~min}$ before initiating the $\mathrm{GaN}$ growth. Nitrogen rich growth conditions were chosen to form self-assembled nanorods that grow along the wurtzite $\mathrm{c}$ axis. Details on the growth can be found in [5].

The all-optical sensor system consists of the NW transducers and a Y-shaped light fiber, which guides the excitation light from a $365 \mathrm{~nm}$ UV power LED to the opto-chemical transducer 
and which collects the longer-wavelength PL emission light for detection in a photomultiplier tube.
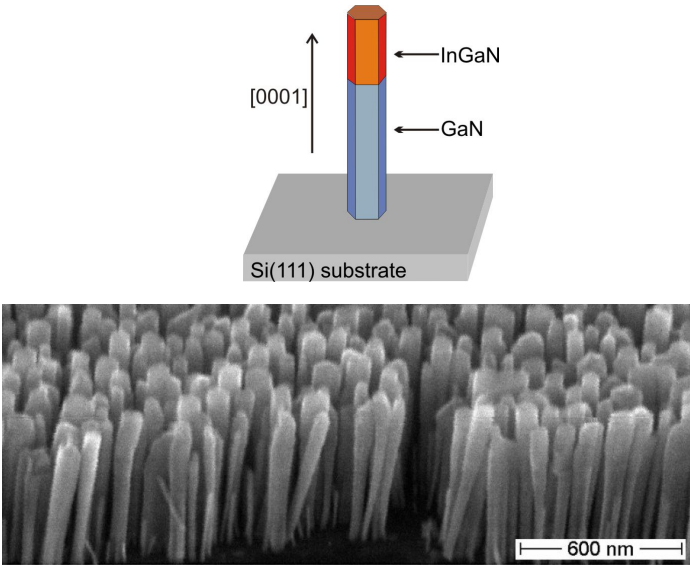

Fig 1. (top) Schematic illustration of the NW structure (not to scale); (bottom) SEM micrograph of $\mathrm{GaN} / \mathrm{InGaN} N$ Ws on Si(111) taken at $45^{\circ}$ inclination. The upper part (InGaN) is slightly conically widened.

\section{Experimental Results}

The NW transducers react with a well pronounced variation in the $\mathrm{PL}$ emission intensity when exposed to small concentrations of hydrogen, methane and acetylene. As an example, Fig. 2 illustrates the response of the optical sensor system to different concentrations of hydrogen as applied at an operation temperature of $30^{\circ} \mathrm{C}$. The optical sensor system is able to clearly resolve $\mathrm{H}_{2}$ concentrations of $20 \mathrm{ppm}$. Although elevated temperatures are necessary for the catalytic dissociation of hydrocarbons at Pt surfaces, a significant hydrocarbon response can already be recognized at room temperature, although with long response and recovery times. Time constants for gas adsorption and desorption significantly decrease as the sensor operation temperature is increased.

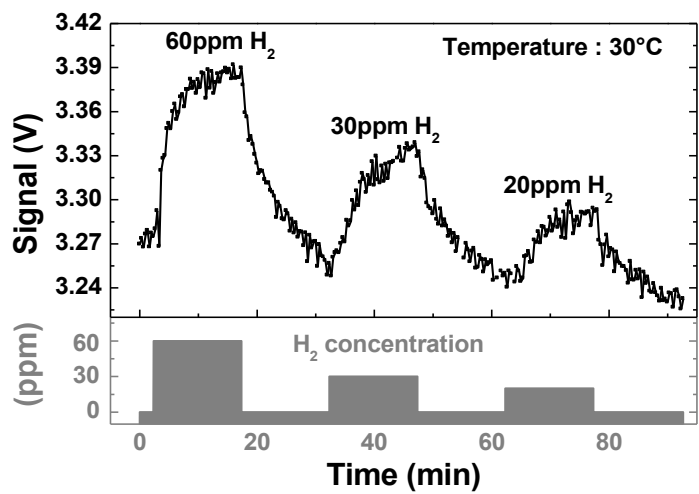

Fig. 2. Photomultiplier output signal in response to decreasing $\mathrm{H}_{2}$ concentrations as observed at a transducer temperature of $30^{\circ} \mathrm{C}$
Fig. 3 summarizes results obtained with the optical sensor system upon different concentrations of acetylene $\left(\mathrm{C}_{2} \mathrm{H}_{2}\right)$ at increasingly higher temperatures.

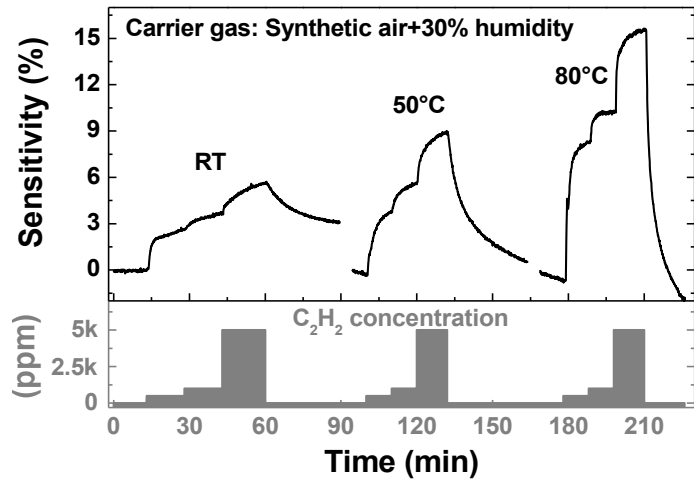

Fig. 3. Sensitivity to three $\mathrm{C}_{2} \mathrm{H}_{2}$ concentrations (500, 1000 and 5000 ppm) as applied at increasingly higher transducer temperatures.

Similar results were obtained when detecting methane $\left(\mathrm{CH}_{4}\right)$, propane $\left(\mathrm{C}_{3} \mathrm{H}_{8}\right)$ and ethylene $\left(\mathrm{C}_{2} \mathrm{H}_{4}\right)$. In general, the relative change in signal intensity and the speed of gas response correlate with the stability of the chemical bonds in the different hydrocarbon molecules.

\section{Acknowledgement}

The presented work was financially supported by the EU FP7 project DOTSENSE (STREP, Grant No. 224212).

\section{References}

[1] I. Lundström, M.S. Shirvamavan, C. Svensson, Appl. Phys. Lett. 26, (1975) 75.

[2] A. Arab, A. Spetz, I. Lundström, Sensors \& Materials 4, (1993) 173.

[3] H. Morkoç, "Nitrides Semiconductors and Devices", Springer Verlag, 1999, pp. 295-339

[4] J. Teubert et. al. "GaN nanodiscs embedded in nanowires as optochemical transducers" Nanotechnology 22275505 (2011)

[5] F. Furtmayr et.al., "Nucleation and growth of GaN nanorods on Si (111) surfaces by plasmaassisted molecular beam epitaxy - The influence of Si- and Mg-doping" J. Appl. Phys 104, 034309 (2008). 